\title{
Comprehensive Theoretical and Experimental Study of Short- and Long-Term Stability in a Passively Mode-Locked Solitonic Fiber Laser
}

\author{
M. Brotons-Gisbert, G. E. Villanueva, J. Abreu-Afonso, G. Serafino, A. Bogoni, M. V. Andrés, and P. Pérez-Millán
}

\begin{abstract}
We demonstrate the short- and long-term stable operation of an all-polarization-maintained Fabry-Pérot cavity passively mode-locked fiber laser. The laser operates in an allanomalous-dispersion solitonic regime. Laser stability is studied by a variety of measurements, which confirm the high stability of the laser in the temporal and spectral-both optical and electrical-domains. Pulse durations of $540 \mathrm{fs}$, period-relative time jitters of $\sim 0.015 \%$, and long-term uninterrumped operation with $0.4 \%$ variation (standard deviation) in the average output power are obtained. The highly stable operation of the laser oscillator was maintained after amplifying the laser output with a conventional EDFA. Pulse durations of $\sim 244$ fs, period-relative time jitters of $\sim 0.019 \%$, and an average output power of $20 \mathrm{~mW}$ were obtained after amplification, while maintaining the $100-\mathrm{dB}$ signal-to-noise ratio of the laser oscillator measured at $500-\mathrm{Hz}$ offset from the fundamental harmonic frequency. The theoretical validation of our experimental results is based on solutions of the Nonlinear Schrödinger Equation. We demonstrate that wavelength and $z$-position dependences of the active medium gain must be taken into account for an accurate correspondence with the experimental properties of the laser.
\end{abstract}

Index Terms-Erbium lasers, fiber lasers, laser stabilization, mode-locked lasers, ultrafast lasers.

\section{INTRODUCTION}

$\mathbf{F}$ EMTOSECOND pulsed lasers with high repetition rates (tens of megahertz to tens of gigahertz) have proven to be suitable optical sources for applications like ultrafast spec-

Manuscript received December 16, 2014; revised May 20, 2015 and June 24, 2015; accepted July 5, 2015. Date of publication July 9, 2015; date of current version August 17, 2015. This work was supported by the Spanish Center for Industrial Technological Development-CDTI (project IDI-20140623), by the Ministerio de Ciencia e Innovación of Spain (project TEC 2008-05490), and by Generalitat Valenciana (programs PROMETEO/2009/077 and GV/2012/121 Grupos Emergentes). The work of M. Brotons-Gisbert was supported in part by the "Atracció de Talent, VLC-CAMPUS" UV-INV-PREDOC13-110538 program of the University of Valencia. The work of P. Pérez-Millán was supported by the Torres Quevedo Grant PTQ-12-05593 of the Spanish Ministry of Economy and Competitiveness, MINECO.

M. Brotons-Gisbert and M. V. Andrés are with the Instituto de Ciencia de los Materiales, Universidad de Valencia, Burjassot, Valencia 46100, Spain (e-mail: mauro.brotons@uv.es; miguel.andres@uv.es).

G. E. Villanueva is with the Nanophotonics Technology Center, Universidad Politécnica de Valencia, Valencia 46022, Spain (e-mail: guivilib@ntc.upv.es).

J. Abreu-Afonso and P. Pérez-Millán are with FYLA LASER SL, Valencia 46980, Spain (e-mail: ppmillan@fyla.com).

G. Serafino is with the Institute of Communication, Information, and Perception Technologies, Pisa 56124, Italy (e-mail: g.serafino@sssup.it).

A. Bogoni is with the National Laboratory of Photonic Networks, Interuniversity National Consortium for Telecommunications, Pisa 56124, Italy (e-mail: antonella.bogoni@cnit.it).

Color versions of one or more of the figures in this paper are available online at http://ieeexplore.ieee.org.

Digital Object Identifier 10.1109/JLT.2015.2455153 troscopy [1], optical biomedicine [2]-[4], and ultrahigh-bit-rate communications, such as wavelength-division multiplexing [5], [6], or photonic-assisted analog-to-digital conversion (PADC) [7], [8]. Some of these applications require extremely high signal quality (e.g., period-relative pulse time jitters of the order of $0.01 \%$, and short-term root-mean-square pulse amplitude fluctuations of the order of $0.1 \%$ in the case of PADC). Passively mode-locked fiber lasers have been demonstrated to be reliable, compact, and cost effective sources of femtosecond pulses [9]. In addition, experimental evidences and theoretical solutions of the Nonlinear Schrödinger Equation (NLSE) of pulse propagation inside the cavity of passively mode-locked fiber lasers demonstrate that certain solitonic solutions provide high-quality signals with extremely low phase and amplitude noise. Typically fiber lasers operate at low repetition rates (in the range of tens of Megahertzs) due to their long laser cavities. However, linear Fabry-Pérot fiber cavities have shown to be an excellent solution for obtaining very-short cavities with high repetition rates [10], [11]. When integrating mode-locked fiber lasers in practical applications, it is indispensable to ensure the long-term and environmental stability of the operational specifications of the laser source. In 2005, Hartl et al. demonstrated that environmental instabilities can be largely eliminated by using polarization-maintaining (PM) fibers in fiber lasers [12]. So far, environmentally robust fully-PM fiber lasers passively mode-locked using different techniques have been reported in a variety of configurations [12]-[17]. Nevertheless, demonstration of long-term stability of ultrafast fiber lasers is commonly avoided or missing in the literature. To our knowledge, these kind of measurements have only been reported by Liu et al. in [16], and Byun et al. in [10]. In [16], Liu et al. demonstrated a highly stable femtosecond all-PM Yb-doped fiber laser, based on the use of an intra-cavity photonic crystal fiber for both oscillator dispersion management and stabilization, and stability measurements of the laser output power during at least $6 \mathrm{~h}$ of operation were presented. In [10], the authors presented a high repetition rate fiber laser passively mode-locked by a saturable Bragg reflector (SBR). The cavity was based on an anomalously dispersive highly Erbium-doped fiber that allowed soliton formation. Long-term stability was addressed by minimizing the thermal damage of the SBR by inserting a few-mm long piece of standard passive fiber between the gain fiber and the SBR in order to avoid the SBR to be in direct contact with the hot core of the active fiber.

In this work, we demonstrate the short- and long-term stability of an all-PM, Fabry-Pérot cavity fiber laser, passively 
mode-locked with a semiconductor saturable absorber mirror (SESAM). An Erbium-doped PM fiber with anomalous dispersion is used as gain medium, which in contrast to [16], makes dispersion management easier than with Ytterbium, since commercially available standard telecom fibers with anomalous dispersion at $1550 \mathrm{~nm}$ can be used, and photonic crystal fibers are not required to compensate normal dispersion. The cavity configuration is simple, maintenance-free and gives rise to selfstarting passive mode-locking in solitonic regime. Short- and long-term stability is achieved by using an intracavity polarizer and by maintaining a fixed linear polarization state throughout the laser cavity. Our setup showed high stability and operated with the same pulsed regime properties over long periods of time (day-scale), insensitive to laboratory environmental fluctuations. Short-term stability is studied through amplitude and phase-noise analysis of the photodetected signal in the temporal and RF-spectral domain. With regard to long-term operation, we carry out real-time measurements of the peak power of individual pulses over long periods of time. In contrast to the methodology in [10] and [16], where only mean power of the laser signal is measured over time, our measurement is a more reliable and rigorous demonstration of uninterrupted long-term pulsed operation. In the optical domain, stability of the optical spectrum (OS) over long-time operation is tested as well. Pulse durations of $540 \mathrm{fs}$, period-relative time jitters of $\sim 0.015 \%$, and maximum variations of $0.4 \%$ in the average output power are obtained. Cavities with free spectral ranges (FSRs) ranging from $30 \mathrm{MHz}$ to $1 \mathrm{GHz}$ have been studied. The theoretical validation of our experimental results is based on solutions of NLSE that take into account wavelength and $z$-position dependence of the gain of the fiber laser active medium. In order to properly model numerically the emission properties of our fiber laser, it has proven crucial and necessary to include in our theoretical model such spectral and $z$-position dependence of the active medium gain. When using this theoretical model, calculated values of width and shape of the laser output in both spectral and temporal domains, match their corresponding experimental results with very high accuracy. The reliability of our numerical method is also confirmed by comparing the experimental and calculated shape and width of the laser pulses, in both optical and spectral domains, after propagating the laser output through different lengths of standard single mode fiber. The high correspondence observed between the experimental and calculated values has motivated us to study numerically the evolution of the spectral and temporal width of the pulses inside the resonator cavity. Differences of $50 \mathrm{fs}$ in the temporal width of the pulses during the stable mode-locked operation of the laser are predicted from numerical results, depending on the output position and the direction of propagation of the light inside the cavity. These results suggest us the possibility of using the numerical method for future cavity engineering.

Finally, the stability of the pulsed signal has also been studied after amplifying the laser output with a commercial erbiumdoped fiber amplifier (EDFA). This amplification process increases the average output power in $15 \mathrm{~dB}$, and broadens the spectral bandwidth from 5.9 to $15.1 \mathrm{~nm}$, while maintaining a signal-to-noise ratio (SNR) of $100 \mathrm{~dB}$ measured at a frequency

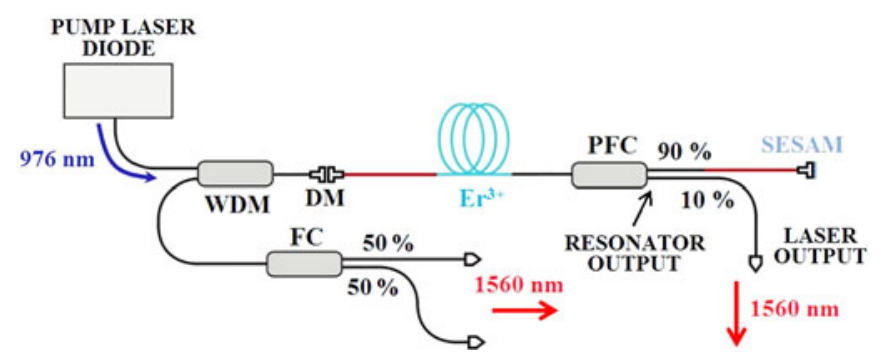

Fig. 1. Experimental setup of the mode-locked, polarization-maintained, Fabry-Pérot cavity fiber laser.

offset of $500 \mathrm{~Hz}$ from the fundamental frequency of the photodetected signal. Temporal pulse durations of $\sim 244 \mathrm{fs}$ are also obtained by dispersion-induced compression of the pulses delivered from the EDFA.

\section{EXPERIMENTAL SETUP}

Fig. 1 shows the laser setup of the mode-locked, PM, FabryPérot cavity fiber laser. A 976-nm laser diode (LD) delivering a maximum output power of $550 \mathrm{~mW}$ was used as pump. Light delivered from the LD was introduced into the linear laser cavity through a dichroic mirror (DM) deposited on the surface of the FC/PC connector of the common port of a standard single-mode 976/1550 nm wavelength division multiplexer (WDM), which couples the pump to the DM. The DM has a reflectance of $99 \%$ at $1550 \mathrm{~nm}$, and a transmittance of $96.5 \%$ at $976 \mathrm{~nm}$. A second uncoated FC/PC connected to a $0.5 \mathrm{~m}$ long, passive, PM fiber (PM1550-HP) was coupled to the coated connector, sandwiching the DM between the two flat connectors. An active fiber was fusion-spliced to the end of the PM1550-HP fiber. This active fiber consisted of a $1 \mathrm{~m}$ long, highly Erbium-doped, PM fiber (Nufern PM-ESF-7/125) with an anomalous dispersion of $\sim 16 \mathrm{ps} / \mathrm{nm} \cdot \mathrm{km}$ at $1550 \mathrm{~nm}$. The end of the active fiber was fusion-spliced to a 90:10 polarizing fiber coupler (PFC) mounted in a $0.88 \mathrm{~m}$ long, passive, PM fiber (Nufern PM980-XP), which presents a measured anomalous dispersion of $13 \mathrm{ps} / \mathrm{nm} \cdot \mathrm{km}$ at $1550 \mathrm{~nm}$. The $90 \%$ port of the PFC was spliced to a $0.5 \mathrm{~m}$ long, FC/APC connectorized PM1550-HP fiber with an estimated anomalous dispersion of $\sim 12 \mathrm{ps} / \mathrm{nm} \cdot \mathrm{km}$ at $1550 \mathrm{~nm}$. A Batop InGaAs SESAM with a modulation depth, saturation fluence and recovery time of $22 \%, 30 \mu \mathrm{J} / \mathrm{cm}^{2}$, and $2 \mathrm{ps}$, respectively, was placed at the end of the PM1550-HP FC/PC connector. The PFC was used both to guarantee single polarization operation and to extract the linearly polarized light from the resonator through its $10 \%$ port. A 50:50 standard single-mode fiber coupler (FC) was fusion-spliced to the 1550-nm port of the WDM, allowing multiple measurements to be carried on simultaneously.

This laser setup gives rise to a repetition rate of $36.77 \mathrm{MHz}$. However, the repetition rate can be tuned to values up to $1 \mathrm{GHz}$ (see Section V) by decreasing the total length of fiber in the cavity.

\section{NUMERICAL MODEL}

In order to model numerically the emission properties of our fiber laser, pulse propagation in the laser cavity is computed by 
solving the NLSE (1) using a standard symmetrized split-step Fourier method algorithm [18]

$$
\frac{\partial}{\partial z} A(z, T)=(\hat{L}+\hat{N}) A(z, T)
$$

NLSE (1), a crucial equation in a fiber transmission system, describes the propagation of the slow-varying envelope $A(z, T)$ of a single polarization of the scalar electric field of an optical pulse normal to its propagation axis. $z$ is the spatial coordinate along the fiber length and $T$ is a group velocity moving-frame time defined as $T=t-\beta_{1} \cdot z$, with $t$ the absolute time and $\beta_{1}$ the inverse of the group velocity. $\hat{L}$ is a linear operator that accounts for gain, losses and dispersion of the optical fiber and $\hat{N}$ is a nonlinear operator that governs the effect of fiber nonlinearities on pulse propagation. These operators are given by

$$
\begin{aligned}
& \hat{L}=-\frac{\alpha}{2}+\frac{g}{2}-\frac{i \beta_{2}}{2} \frac{\partial^{2}}{\partial^{2} T}+\frac{\beta_{3}}{6} \frac{\partial^{3}}{\partial^{3} T}, \\
& \hat{N}=i \gamma|A(z, T)|^{2} .
\end{aligned}
$$

In (2), $\alpha$ is the fiber loss coefficient, $g$ is the pump and wavelength-dependent gain (4), and $\beta_{n}$ are the $n$-order groupvelocity dispersion parameters. In (3), we have only considered self-phase modulation (SPM) through the nonlinear parameter $\gamma$ of the fiber, which is given by the nonlinear refractive index $n_{2}$ of the optical fiber and the effective area $A_{\text {eff }}$ of the laser mode in the fiber core according to $\gamma=2 \pi n_{2} /\left(\lambda_{0} \cdot A_{\text {eff }}\right)$. More complex nonlinear effects such as stimulated Raman scattering and self-steepening are neglected in our simulations.

The fiber laser simulation strategy consists of the propagation of the optical field envelope in consecutive round-trips. In one round-trip, the optical pulse is propagated through the different fiber sections of the linear cavity by solving equation (1) in each section, and the wavelength-dependent effects of lumped optical elements (the DM, the PFC and the SESAM) are discretely applied. As a round-trip along the cavity is completed, the resulting pulse is injected into the first section of the cavity, and a new round-trip is computed. This process is repeated, and the output is extracted after convergence, which is checked by stabilization of parameters like pulse peak power or pulsewidth. This convergence is generally reached in few hundreds of round-trips.

Gain in the laser is modeled after equation (70) explained by Digonnet in [19], which accounts properly for saturation effects in the active medium. In order to extend the utility of (70) in [19] to a more realistic scénario, we have considered wavelength and $z$-position dependence of parameters in the gain equation, which takes the following form:

$$
g(\lambda, z)=\Gamma N_{t} \sigma_{e m}(\lambda) \frac{\frac{P_{P}(z)}{P_{P}^{t h}}-\frac{\sigma_{\text {abs }}(\lambda)}{\sigma_{e m}(\lambda)}}{1+\frac{P_{P}(z)}{P_{P}^{\text {th }}}} \frac{1}{1+\frac{P_{S}}{P_{\text {sat }}^{*}(z)}},
$$

where $\Gamma$ and $N_{t}$ are the estimated overlap factor between mode field and erbium dopant distribution, and the total ion density, respectively. Parameters $\sigma_{\mathrm{abs}}(\lambda)$ and $\sigma_{e m}(\lambda)$ represent the wavelength-dependent absorption and emission cross-sections of the active fiber. $P_{s}$ denotes the average pulse power, calculated as $P_{s}=E_{P} / T_{R}$, with $E_{P}=\int_{-\infty}^{\infty}|A(z, T)|^{2} d T$, and $T_{R}$

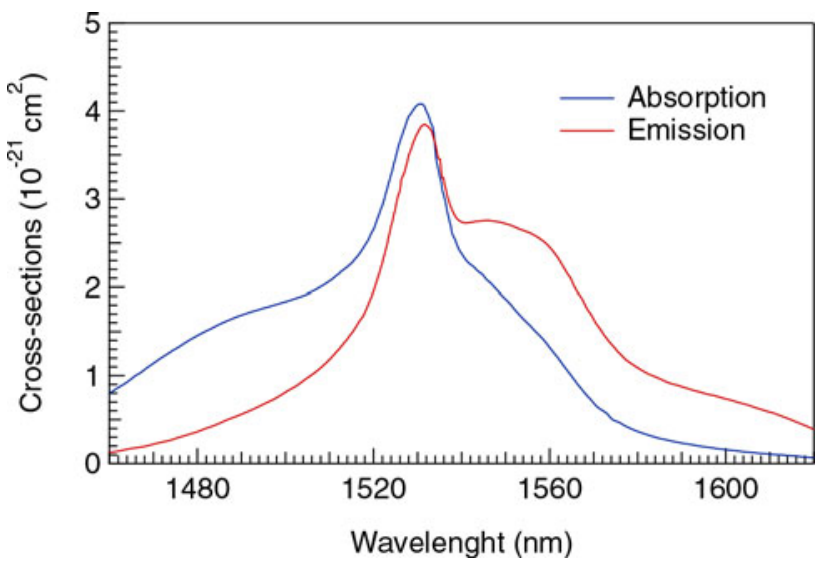

Fig. 2. Wavelength-dependent absorption and emission cross-sections of the active fiber used in the numerical calculations, estimated from technical specifications.

is the cavity round-trip time. $P_{\text {sat }}, P_{p}^{\text {th }}$ and $P_{\text {sat }}^{*}$ are the intrinsic saturation power of the active medium, the pump power threshold (the pump power for which the ground and upper populations are equal) and the effective saturation power of the fiber, which are given by

$$
\begin{aligned}
P_{\text {sat }} & =h \nu_{0} A_{0} \frac{1}{\tau\left(\sigma_{\text {abs }}\left(\nu_{0}\right)+\sigma_{e m}\left(\nu_{0}\right)\right)} \\
P_{P}^{\text {th }} & =\frac{h \nu_{P} A_{P}}{\sigma_{\text {abs }}\left(\nu_{P}\right) \tau}, \\
P_{\text {sat }}^{*}(z) & =P_{\text {sat }}\left(1+\frac{P_{P}(z)}{P_{P}^{\text {th }}}\right),
\end{aligned}
$$

with $P_{P}(z)$ being the estimated effective pump power available after a length $z$ of active fiber, and $\tau$ being an average fluorescence lifetime characterizing the ${ }^{4} I_{(13 / 2)}-{ }^{4} I_{(15 / 2)}$ manifold of Erbium transition. We have assumed that this effective pump power varies along the active fiber length as $P_{P}(z)=P_{P}(0) \cdot \exp \left(-\alpha_{P} \cdot z\right)$, with $P_{P}(0)$ the pump power at the entrance of the active fiber, and $\alpha_{P}$ a parameter that accounts for the fiber absorption at pump wavelength. Parameters $h, \nu_{P(0)}$, and $A_{P(0)}$ represent the Planck constant, the pump (central carrier) frequency and the mode effective area of the active fiber at pump (central carrier) frequency. Fig. 2 shows the wavelength-dependent emission and absorption cross-sections of the active fiber estimated from its technical specifications and used in our numerical calculations.

Considering in our numerical model such spectral and zposition dependence of the active medium gain has proven to be crucial and necessary in order to properly model numerically the emission properties of our fiber laser. When including such dependences of the active medium gain, not only calculated values of width and shape of the laser output match their corresponding experimental results with very high accuracy (in both spectral and temporal domains), but also laser threshold and laser output powers are very similar to those obtained experimentally. By contrast, not considering such dependences of the active medium gain gives rise to very unrealistic values of the laser threshold and laser output powers (see Section IV, Fig. 6). 
TABLE I

VAlues of Some of the PARAMETERS USED IN THE SimUlations

\begin{tabular}{lc}
\hline \hline Parameter & Value \\
\hline SESAM non-saturable losses & $15 \%$ \\
SESAM recovery time $\left(\tau_{S A}\right)$ & $2 \mathrm{ps}$ \\
SESAM saturation fluence & $30 \mu \mathrm{J} / \mathrm{cm}^{2}$ \\
SESAM modulation depth $(\Delta \mathrm{R})$ & $22 \%$ \\
Pump wavelength & $976 \mathrm{~nm}$ \\
Signal wavelength & $1561 \mathrm{~nm}$ \\
Effective area of the laser mode $\left(A_{\text {eff }}\right)$ & $62.2 \mu \mathrm{m}^{2}$ \\
Average second-order dispersion $\left(\beta_{2}\right)$ & $-17.4 \cdot 10^{-3} \mathrm{ps}^{2} / \mathrm{m}$ \\
Average third-order dispersion $\left(\beta_{3}\right)$ & $0 \mathrm{ps}^{3} / \mathrm{m}^{-1}$ \\
Average nonlinear parameter $(\gamma)$ & $1.6\left(\mathrm{~W} \cdot \mathrm{km}^{-1}\right.$ \\
\hline \hline
\end{tabular}

Regarding the SESAM effect in the numerical calculation, it was evaluated as an insertion loss dependent on the pulse intensity as done in [20]:

$$
\begin{aligned}
& A(\text { after } S A, T)= \\
& =A(\text { before } S A, T) \cdot \exp \left(-\frac{q(A(z, T))+q_{\text {non }}}{2}\right) .
\end{aligned}
$$

To take into account the temporal response of the absorber, the SESAM losses obey the expression [20]

$$
\frac{\partial q(A(z, T))}{\partial T}=-\frac{q-q_{0}}{\tau_{S A}}-q \frac{|A(z, T)|^{2}}{E_{S A}} .
$$

In (9), $\tau_{S A}, q_{0}$ and $E_{S A}$ are the recovery time, the saturable loss and the saturation energy of the saturable absorber mirror, respectively. Saturable absorption also includes an amplitudeindependent term $q_{\text {non }}$ to account for the non-saturable losses, which is directly added to the saturable term as (8) shows.

Random noise input was employed as initial seed in all our simulations. Computed pulse temporal wavefunctions and OSs are extracted from calculations.

Table I shows the values of some of the parameters used in our simulations. It is worth mentioning that we have observed that the third-order dispersion coefficient is not very important for an adequate description of our laser output characteristics. The relatively small average pulse power inside the cavity along with the fact that the laser operation wavelength is far enough from the zero-dispersion wavelength, allowed us to consider an average third-order dispersion value of $0 \mathrm{ps}^{3} / \mathrm{m}$.

\section{NUMERICAL AND EXPERIMENTAL MODE-LOCKING RESULTS}

Fig. 3(a) shows the calculated stable mode-locked pulse formation in an anomalous net dispersion solitonic regime corresponding to the experimental setup described in Section II. Evolution of the simulated peak power and time full-width at half maximum (FWHM) of the pulses are depicted in the inset. Fig. 3(b) shows the OS evolution corresponding to the modelocked pulse formation shown in Fig. 3(a). The inset in Fig. 3(b) presents the peak power and FWHM evolution of the OS. Stability of both peak power and FWHM in the temporal and spectral domains is reached after few hundreds of round-trips, when the (a)

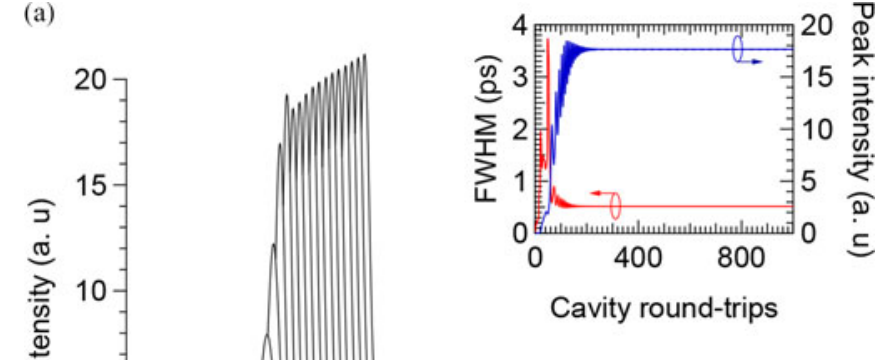

(b)

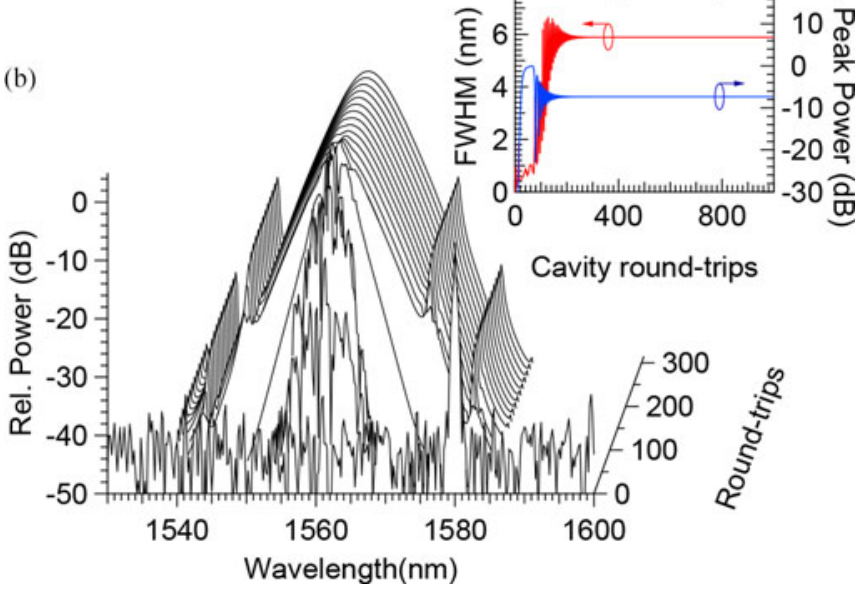

Fig. 3. (a) Calculated stable mode-locked pulse formation in an anomalous net dispersion solitonic regime corresponding to the setup described in Section II. The inset shows the simulated pulse peak power and pulse FWHM evolution. (b) Calculated evolution of the OS during the mode-locked pulse formation shown in (a). The inset shows the simulated spectral FWHM and peak power evolution.

initial random noise input is mode-locked into a stable $517 \mathrm{fs}$ FWHM pulse with a $5.9 \mathrm{~nm}$ spectral bandwidth.

Fig. 4 shows the calculated evolution of the temporal and spectral FWHMs of the pulse at different positions along the resonator cavity, corresponding to a round-trip when the laser is stable mode-locked. Squares and circles represent the forward and backward direction of pulse propagation along the cavity, respectively. As can be seen in this figure, differences of 50 fs in the temporal pulse FWHM during a stable mode-locked round-trip, are predicted by numerical calculations, depending on the cavity position and the propagation direction. These results suggest the possibility of using the numerical calculations for future cavity engineering to optimize the laser output pulse properties by selecting properly the position and propagation direction of the cavity output.

Our experimental setup worked in a mode-locked solitonic regime for pump powers over a pump threshold of $90 \mathrm{~mW}$. This mode-locked regime was always self-starting and insensitive to laboratory environmental fluctuations. Once the laser was mode-locked, it could not be unstabilized by, for instance, moving, pressing or tilting the fiber and components of the laser. The 


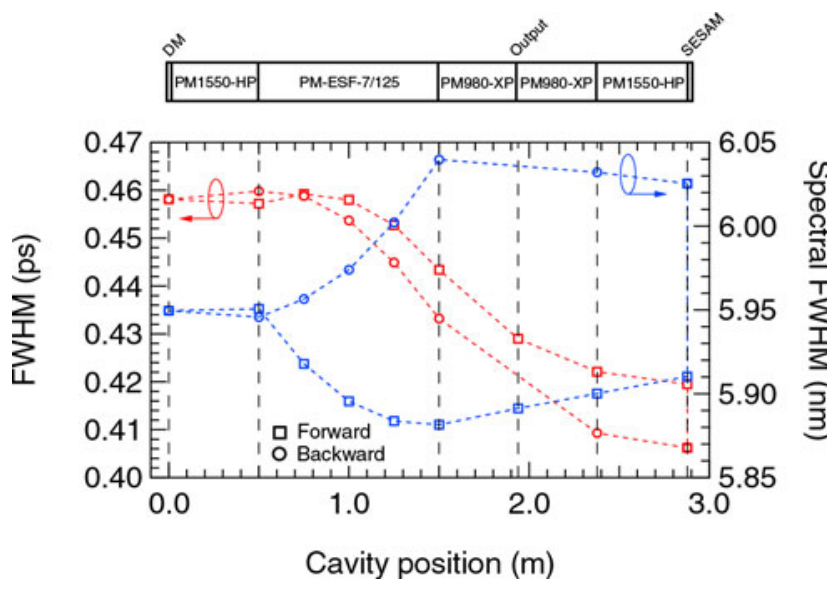

Fig. 4. Calculated evolution of the temporal and spectral FWHMs of the pulse at different positions along the resonator cavity for a stable mode-locked round-trip.

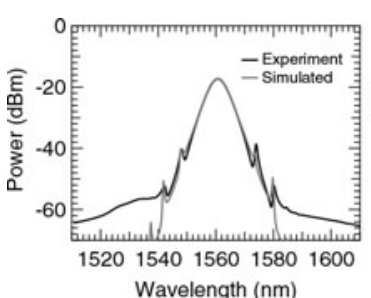

(a)

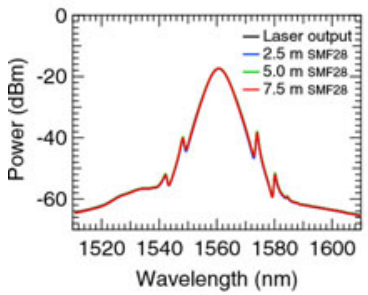

(c)

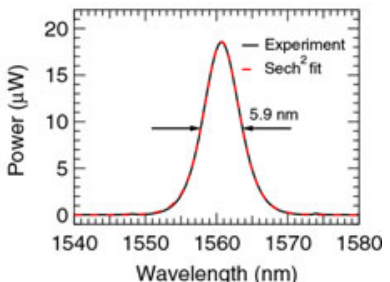

(b)

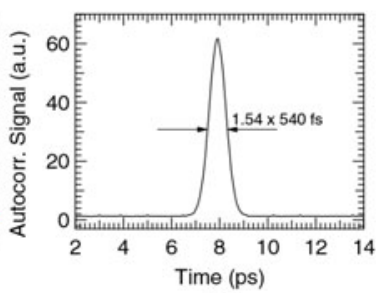

(d)
Fig. 5. (a) Experimental and simulated output OS of the mode-locked fiber laser in logarithmic scale. (b) Experimental output OS in linear scale and its $\operatorname{sech}^{2}$ fit. (c) Output optical spectra measured after propagating the laser output through different lengths of SMF28 fiber. (d) Autocorrelation trace of the laser output.

pump could be switched off and on repeatedly several times and the mode-locking regime always restored itself. Fig. 5(a) shows the measured OS (black line) corresponding to a cavity with a repetition-rate of $36.77 \mathrm{MHz}$ and an anomalous net dispersion of $\sim 0.039 \mathrm{ps} / \mathrm{nm}$, taken at $120 \mathrm{~mW}$ of pump power. This measurement was carried out at the laser output, i.e., after propagating the resonator output through a $2 \mathrm{~m}$ long PM980 passive fiber. The spectrum is centered at around $1561 \mathrm{~nm}$, with a FWHM optical bandwidth of $5.9 \mathrm{~nm}$. Kelly sidebands indicative of solitonic regime can be observed. The laser operation wavelength is not tunable. However, given that the laser operation wavelength is determined by the spectral dependence of the ratio between the net gain and the cavity losses, it might be possible to tune the operation wavelength of the laser by modifying the spectral dependence of the cavity losses. No appreciable changes in the OS were observed during the experiments, which was indicative of the high long-term stability of the pulsed regime. The OS

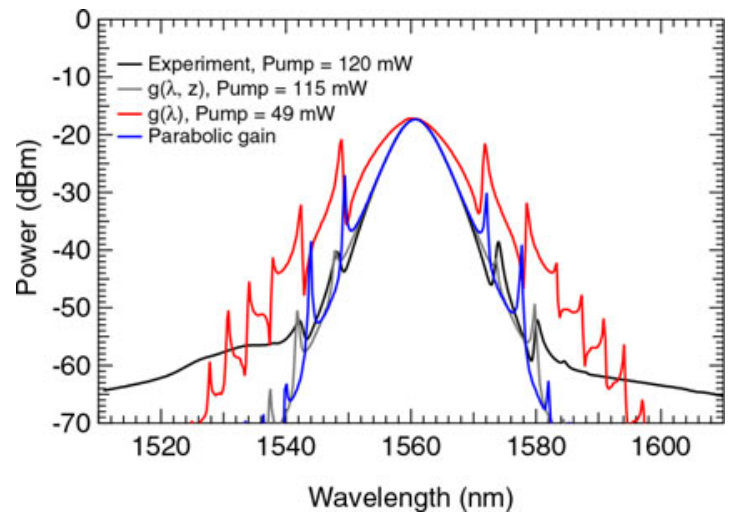

Fig. 6. Comparison of the numerical optical spectra calculated using different gain models.

remained very stable not only after few-hours long continuous operation but also every time we switched on the laser. The high stability of the OS of this laser during long-term operation relies on both all-in-PM-fiber and use of an intra-cavity polarizer. Fig. 5(a) also shows the calculated output OS (grey line) of the corresponding experimental setup, obtained for a nominal pump power of $115 \mathrm{~mW}$. A high correspondence between simulated and experimental spectra is observed, with a FWHM deviation of less than $1 \%$. As expected, Kelly sidebands are also observed in this theoretical calculation, appearing at wavelengths matching nearly exactly the experimental ones. The very small differences in the Kelly sidebands wavelength observed in the measured and simulated spectrum indicate a very accurate approximation between experimental and theoretical values of cavity length and cavity net dispersion. Random noise input was employed as initial seed in the simulation. Fig. 5(b) shows the measured OS in linear scale and its hyperbolic secant squared $\left(\operatorname{sech}^{2}\right)$ fit.

Fig. 6 shows the effects of including the spectral and $z$ position dependence of the active medium gain in the numerical results. In this figure we compare the measured OS (black line) with the numerical optical spectra calculated by using three different gain models. $g(\lambda, z)$ (grey line) represents the OS obtained when considering the gain model described by equation (4) in Section III, for a nominal pump power of $115 \mathrm{~mW}$. As it has already been discussed previously [see Fig. 5(a)], this gain model gives rise to a high correspondence between the simulated and experimental spectra. It is worth to notice that also the nominal pump power used in the calculation agrees well with the pump power measured experimentally. $g(\lambda)$ (red line) represents the numerical OS obtained when the gain of the active medium is modeled by equation (70) in [19], which does not take into account the z-position dependence of the gain in the active fiber. Such numerical OS has been calculated for a nominal pump power of $49 \mathrm{~mW}$, resulting in a higher FWHM optical bandwidth than the experimental OS. This behavior indicates that not considering the z-position dependence of the pump power gives rise to unrealistic values of the nominal pump power when compared with the experimental one. Moreover, if the nominal pump power is increased above $49 \mathrm{~mW}$, the calculated mode-locked output pulse in the temporal domain 


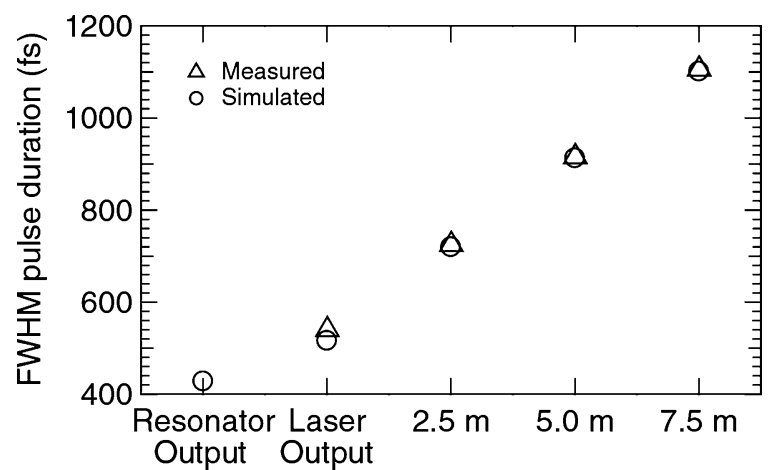

Fig. 7. Measured and calculated FWHM pulse durations obtained at the laser output and after propagating the laser output through a $2.5,5.0$ and $7.5 \mathrm{~m}$ long standard single mode fiber (SMF28).

splits into multiple pulses instead of becoming a more intense single pulse. Finally, the blue line represents the OS calculated when the gain medium is modeled assuming a parabolic approximation [20], which does not present an explicit dependence on the nominal pump power. The numerical OS obtained using this gain model reproduces well the FWHM optical bandwidth of the experimental OS. However, the spectral positions of the Kelly sidebands predicted by this model do not agree with the experimental ones as accurately as the ones obtained using the $g(\lambda, z)$ gain model.

We have also measured the OS after propagating the laser output through different lengths of standard single mode fiber (SMF-28). Fig. 5(c) shows the output OS obtained in each one of these measurements. As can be seen in this figure, the OS remains unaltered, as expected in absence of nonlinear effects. A sech ${ }^{2}$ OS with a $5.9 \mathrm{~nm}$ FWHM implies a Fourier-transformlimited FWHM pulse duration of $430 \mathrm{fs}$.

The pulse intensity profile of the laser output has also been measured by use of an autocorrelator. For the autocorrelation measurement, the laser output was introduced in the autocorrelator through a dispersion shifted fiber with a near-to-zero dispersion at $1560 \mathrm{~nm}$. Fig. 5(d) shows the autocorrelation trace measured at the laser output. The pulse FWHM of the autocorrelation profile is measured to be $834 \mathrm{fs}$, which when considering a sech ${ }^{2}$ decorrelation factor, yields a $540 \mathrm{fs}$ FWHM pulse duration. The difference between the transform-limited temporal FWHM obtained from the experimental OS (430 fs) and the FWHM pulse duration obtained from the autocorrelation measurement (540 fs), has its origin in the widening of the output pulse when it is propagated along the $2 \mathrm{~m}$ long PM980 fiber that exists between the resonator output and the laser output (see Fig. 1). Fig. 7 illustrates the FWHM pulse durations of the laser output after propagating it through different lengths of standard single mode fiber (SMF-28), obtained from autocorrelation measurements. As can be seen in this figure, the output pulse broadens significantly as it is propagated through just a few meters of standard fiber. This behavior has its origin in the diminution of the pulse's power after the 90:10 output coupler. After this output coupler, the pulses no longer have enough peak power for the nonlinear effects to balance the fiber dispersion and the pulses broaden. Fig. 7 also shows the corresponding FWHM pulse durations through simulated propagation of the

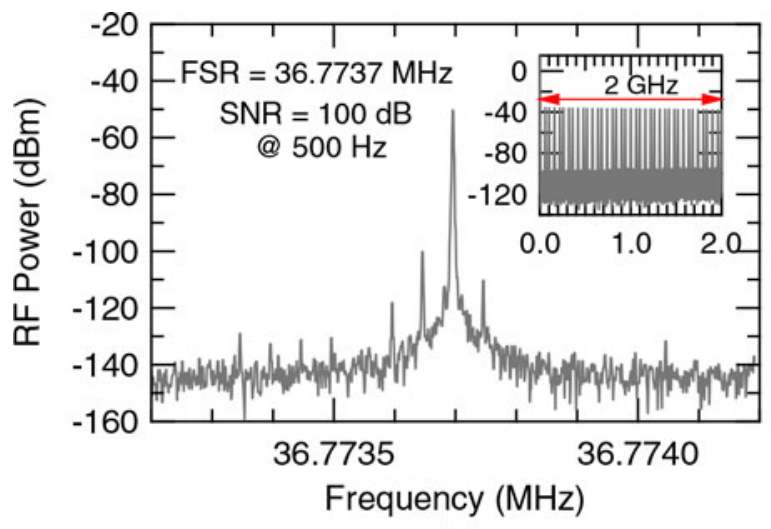

(a)

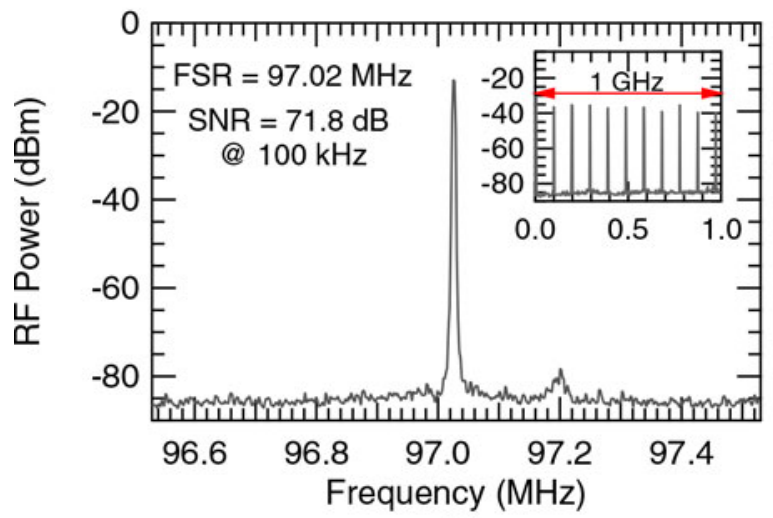

(b)

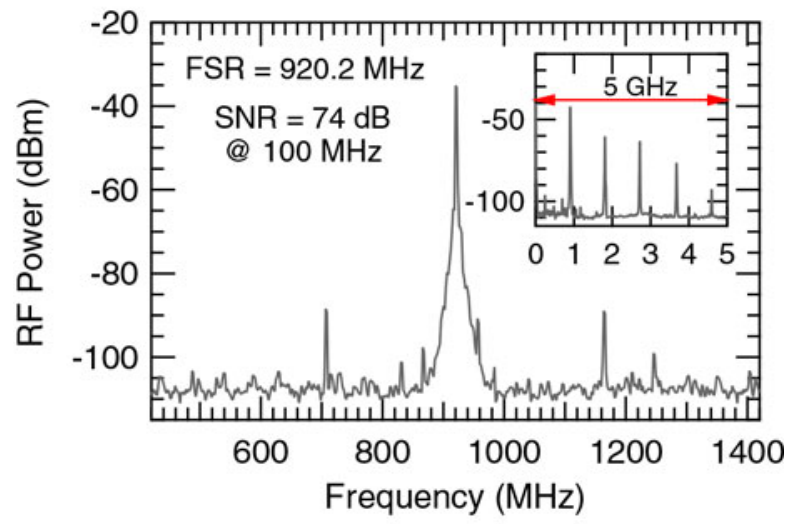

(c)

Fig. 8. RF spectra of the photodetected fundamental harmonic of the modelocked laser output corresponding to setups with FSRs of $36.77 \mathrm{MHz}$ (a), 97.02 $\mathrm{MHz}$ (b), and $920.16 \mathrm{MHz}$ (c). The insets show the corresponding spectra with wider measurement spans.

pulses obtained by our numerical calculation at the resonator output. As shown in this figure, numerical calculations give a 429 and 517 fs FWHM pulse durations at the resonator and at the laser output, respectively. The time-bandwidth product at the resonator output, obtained from the numerical simulations, is calculated to be $\sim 0.319$, which is very close to the Fouriertransform limit of 0.315 for $\mathrm{sech}^{2}$ pulses.

\section{ShORT-Term Stability Results}

In order to study the short-term stability of the laser, RF measurements of the photodetected laser output were carried out. In Fig. 8(a) we show the RF spectrum of the photodetected 


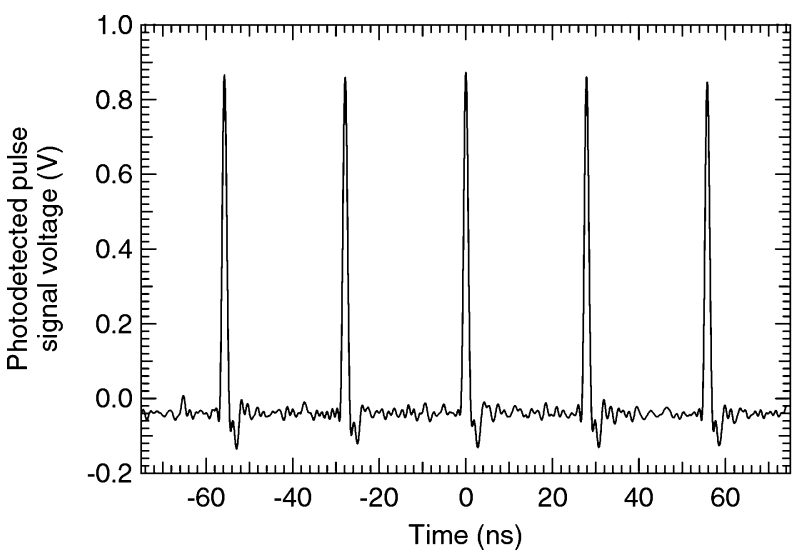

Fig. 9. Oscilloscope pulse train of the photodetected signal of the laser for a cavity with $36.77 \mathrm{MHz}$ FSR.

fundamental harmonic of the mode-locked laser (MLL) output corresponding to a setup with a FSR of $36.77 \mathrm{MHz}$, with a SNR of $100 \mathrm{~dB}$ at $500 \mathrm{~Hz}$ offset from the fundamental frequency. The measurement was carried out with a $1 \mathrm{kHz}$ frequency span and $1 \mathrm{~Hz}$ resolution bandwidth. Noise peaks observed at both sides of the fundamental harmonic correspond to multiples of $50 \mathrm{~Hz}$. They are not part of the laser signal, but spurious interferences of the electric net. The inset shows the corresponding spectrum with a $2 \mathrm{GHz}$ span. Fig. 8(b) and (c) show the RF spectra of the photodetected fundamental harmonic of the laser output corresponding to setups with FSRs of 97.02 and $920.2 \mathrm{MHz}$, respectively. As can be seen in these figures, the laser setup presented in Section II can be scaled to achieve FSRs up to $\sim 1 \mathrm{GHz}$ while mantaining a good short-term stability.

Oscilloscope pulse train traces of the photodetected laser output were also measured in order to check the short-term stability of our experimental laser setups. Fig. 9 illustrates an example of recorded oscilloscope pulse train of a cavity with a FSR of 36.77 MHz. Photodetection in this measurement was performed with a photodiode (PD) of $2.5 \mathrm{GHz}$ bandwidth. A standard deviation of $1.2 \mathrm{mV}$ in the peak amplitude was obtained from 2350 oscilloscope samples of the photodetected signal, which corresponds to peak amplitude fluctuations of $0.14 \%$. This is an upper value for the actual short-term optical peak amplitude noise, since it includes the contribution of electronic noise from photodetector and oscilloscope.

We also investigated the performance of the MLL in terms of phase noise in order to study its short-term stability in more depth. Phase fluctuations can be assessed by measuring the phase noise power spectral density (PSD) $S_{\Delta \varphi}(f)$ of the laser electric spectrum at a given multiple $f_{c}$ of its fundamental repetition frequency, and then by calculating the phase jitter $\Delta \varphi$. This parameter can be obtained by applying the formula [21]:

$$
\Delta \varphi=\sqrt{\int_{f_{c}}^{\infty} S_{\Delta \varphi}(f) d f .}
$$

In a real measurement, the upper integration limit will not be infinite in (10); similarly, the lower integration limit is not the exact frequency $f_{c}$ of a mode of the electrical spectrum. Indeed,

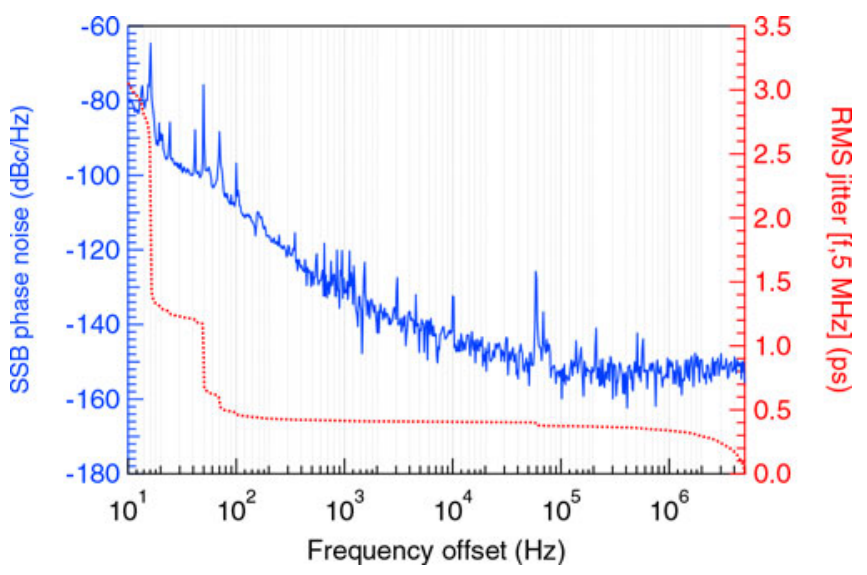

Fig. 10. Single sideband (SSB) phase noise profile corresponding to the setup presented in Section II measured at the laser fundamental repetition frequency $36.77 \mathrm{MHz}$. Figure also shows the $\Delta \tau$ time jitters integrated from $5 \mathrm{MHz}$ progressively down to $10 \mathrm{~Hz}$.

the integration usually starts from a frequency offset imposed by the needed coherence time interval which, in turn, depends from the particular application of the laser. A more commonly used parameter for evaluating the laser stability is the time jitter $\Delta \tau$, simply given by:

$$
\Delta \tau=\frac{\Delta \varphi}{2 \pi f_{c}} .
$$

Therefore, to assess the proposed laser phase stability, we measured its time jitter at the laser fundamental repetition frequency $36.77 \mathrm{MHz}$. To this aim, the laser output has been photodetected by a PD with an electric bandwidth of $2 \mathrm{GHz}$, without optical or electrical amplification, and fed into an Agilent E5052A signal source analyser for measuring its phase noise PSD. In Fig. 10, the phase noise profile corresponding to the setup presented in Section II is reported. The phase noise curve depicted in Fig. 10 is an unilateral spectrum, hence the formula applied for determining $\Delta \tau$ has been

$$
\Delta \tau=\frac{1}{2 \pi f_{c}} \sqrt{2 \int_{f_{L}}^{f_{H}} S_{\Delta \varphi}(f) d f .}
$$

The selected upper integration limit $f_{H}$ is $5 \mathrm{MHz}$ from $f_{c}$, set by the measurements instrument which, for that offset frequency value, entered in its noise floor region. The time jitter integrated from $5 \mathrm{MHz}$ progressively down to $10 \mathrm{~Hz}$ is also shown in Fig. 10 as a red dashed line, resulting in integrated time jitters as low as 3.06 ps (from $10 \mathrm{~Hz}$ ), $472.3 \mathrm{fs}$ (from $100 \mathrm{~Hz}$ ), and $414.4 \mathrm{fs}$ (from $1 \mathrm{kHz}$ ), which correspond to phase jitters of $7.0 \cdot 10^{-4}$, $1.1 \cdot 10^{-4}$, and $9.0 \cdot 10^{-5} \mathrm{rad}$, respectively. As can be seen from equation (11), the time jitter of a laser is directly proportional to its repetition temporal period (inversely proportional to its fundamental repetition frequency). A more intuitive parameter to compare the stability of a laser is the time jitter relative to its repetition temporal period. Such parameter can be obtained by dividing phase noise jitter by $2 \pi$ [see equation (11)]. Measured phase jitters give rise to period-relative time jitter values of $0.11 \%$ (from $10 \mathrm{~Hz}$ ), $0.017 \%$ (from $100 \mathrm{~Hz}$ ), and $0.015 \%$ 
(from $1 \mathrm{kHz}$ ). Our measured value of the period-relative time jitter integrated over the $[1 \mathrm{kHz}-5 \mathrm{MHz}]$ frequency interval is of the same order of magnitude than the one resulting from the MLL setup presented by Byun et al. in [10], which can be estimated to be $\sim 0.02 \%$ from their reported time jitter value of $22 \mathrm{fs}$ integrated over the [ $1 \mathrm{kHz}-10 \mathrm{MHz}$ ] frequency interval and the $967 \mathrm{MHz}$ laser fundamental repetition frequency. Although this value is obtained integrating over a frequency interval broader than ours, it can be used to compare the stability of both lasers due to the low phase noise PSD in the [5-10 $\mathrm{MHz}$ ] frequency interval.

As a matter of fact, the jitter of the pulses is comparable to their timewidth. This will be an irrelevant temporal noise in most of the envisioned applications in ultrafast spectroscopy, bioimaging and wavelength-division-multiplexing. In contrast, such jitter will determine performance limits in optical sampling applications, such as photonic analog to digital conversion.

Applications of MLLs in ultrafast spectroscopy rely either on the comb-like spectral shape of the MLLs [1] or on pulse-probe temporal measurements where excitation and measure pulses are synchronized individually. In the first case, the limiting factor is mainly the spectral resolution of the spectrographs, e.g., $0.001 \mathrm{~nm}$ in very good spectrographs, far over the linewidth of the MLL optical harmonics (typ. tens of Hz), which will be altered by only a few tens of hertz by time jitters comparable to pulse widths of hundreds of fs. In the second case, the main limiting factor is the time response of the probe, which being individually synchronized with the pump, is not altered by the jitter between pump train pulses. Bioimaging applications of MLLs rely mainly on nonlinear optical responses of tissues or fluorophores [2], [3], which depend on the intrinsically high peak powers of MLL optical pulses. Images are recorded by averaging such optical responses, hence a time jitter comparable to a pulse time width of hundreds of fs will be irrelevant. In this case, the relevant parameters are the peak power and average power stabilities. Multiphoton microscopy systems typically require $<1 \%$ average power long-term stability (see Section VI). With regard to wavelength-division-multiplexing [6], optical harmonics of the comb-like spectrum shape of the MLLs are suggested as optical carriers. As in spectroscopy applications, spectral resolution of these sources is well over the resolution of the filters used to interleave the carriers. And the optical phase noise corresponding to a time jitter comparable to pulse width of hundreds of fs is well below the phase noise of conventional optical carriers (e.g., diode lasers). The most plausible utility of MLLs as tools for analog to digital conversion is a hybrid ADC architecture, where sampling is performed optically and quantization is performed electronically [7]. In this case, the sampling time can be equal to the optical pulse width and the sampling jitter is equal to the MLL jitter. Therefore, a pulse jitter comparable to the pulse width of the optical source implies that the maximum sampling jitter of an ADC based on the MLLs presented in this article will be approximately $500 \mathrm{fs}$.

In principle, this limit could be overcome using the compressed pulses at the output of the amplifier presented in Section VII, but the pedestal of the pulses will presumably limit the sampling jitter of the ADC performance to an approximately (a)

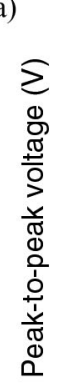

(b)

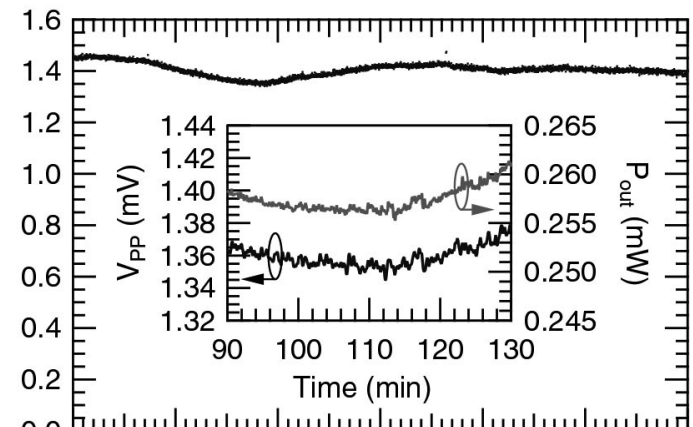

(c)
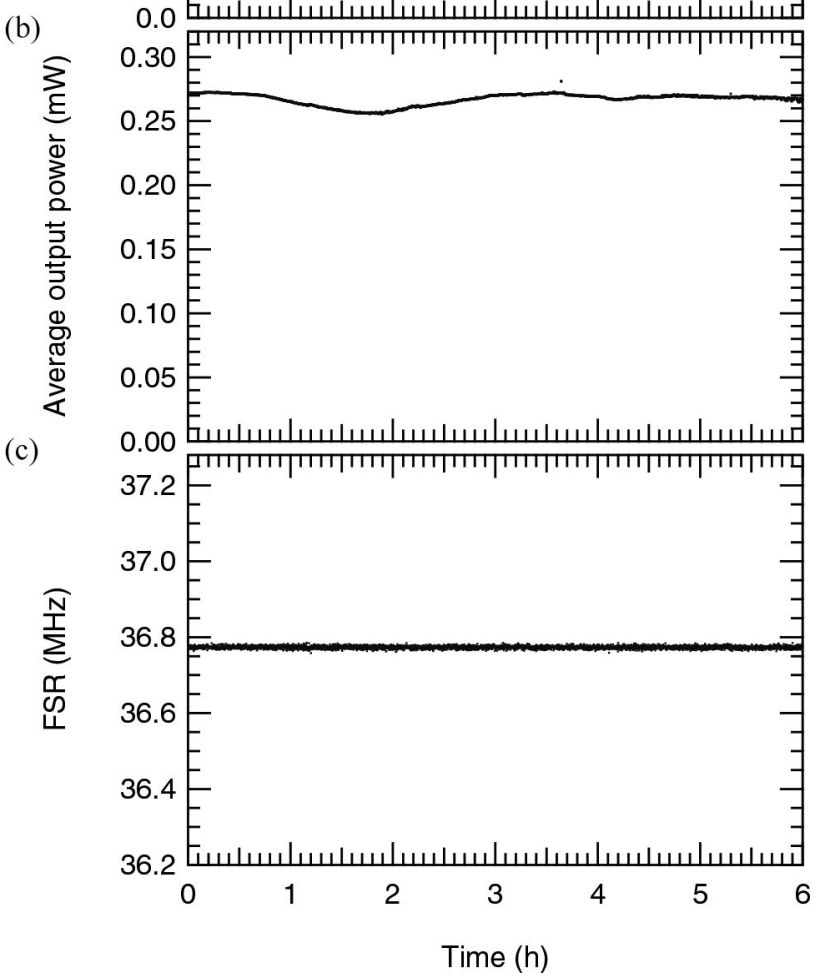

Fig. 11. Evolution of the peak-to-peak voltage of the individually photodetected laser output pulses (a), average output power (b), and FSR (c) of the MLL during 6-h long continuous operation.

equivalent value of the limit of $500 \mathrm{fs}$ imposed by the optical jitter of the pulses. The very short delay between pedestal and principal peaks of the pulse compared to typical times of physical phenomena associated to applications in spectroscopy, bioimaging and wavelength division multiplexing discussed above indicate that this distortion of the pulse will not limit fundamentally the applications envisioned in such fields.

\section{LONG-TERM STABILITY RESULTS}

As described in Section IV, our laser presents high long-term stability in the optical domain, working in a self-starting modelocked solitonic regime, that is insensitive towards mechanical disturbances and laboratory environmental fluctuations. This long-term stability has also been studied in the temporal domain by measuring the average output power, the peak power of the individually photodetected laser output pulses, and the oscillator repetition rate during several-hours long continuous operation. For a launched pump power of $160 \mathrm{~mW}$, the average 


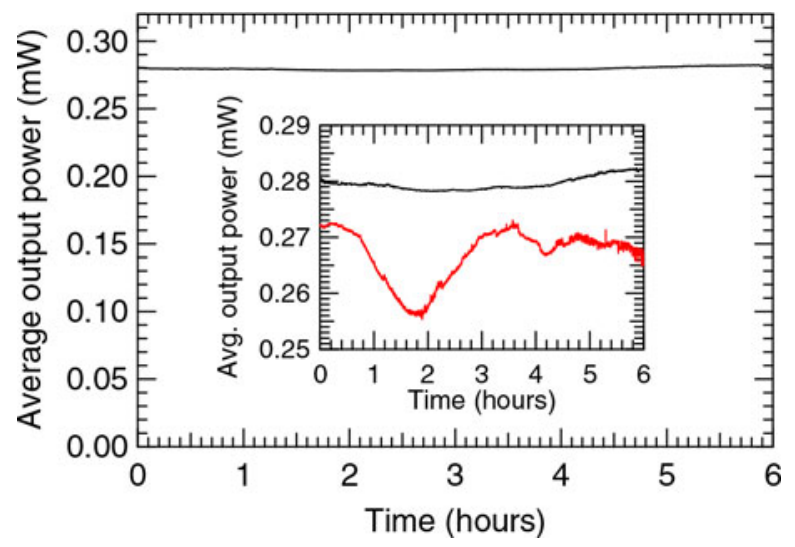

Fig. 12. Evolution of the average output power of the MLL during 6-h long continuous operation after incorporating the TEC control. The inset shows a comparison of the average output power measured for the setup with (black line) and without (red line) the TEC control.

output power was measured using a power meter THORLABS PM100D with a $1.2 \mathrm{GHz}$ InGaAs photodetector head, with a sampling rate of one sample per $30 \mathrm{~s}$. The peak power of the photodetected peaks and the oscillator repetition rate were measured using a $2.5 \mathrm{GHz}$ Agilent Infiniium 90000 real-time oscilloscope after photodetecting the laser output with a PD of $2.5 \mathrm{GHz}$ bandwidth, with the same sampling rate. Fig. 11(a) and (b) show the evolution of the peak power of the individual laser pulses and the average output power corresponding to the setup presented in Section II, respectively. A standard deviation of $<1.8 \%$ in both photodetected peak power and average output power is obtained after 6-h long continuous operation. The main contribution to such deviation is believed to be due to slow variations of the emitted wavelength of the pump diode (since in our setup the pump diode lacks of precise TEC control), yielding slow variations of the fiber laser cavity gain. As shown in the inset of Fig. 11(a), the peak power of the photodetected pulses evolved proportionally to the average output power as function of time, indicating that the laser output was a clean pulse train, free of other optical contributions, such as continuous wave emission, secondary spurious optical harmonics or other optical noise sources (e.g., ASE). In this case, unlike the case of short-term amplitude noise measurements, the peak-to-peak amplitude fluctuations recorded in long-term measurements of Fig. 11 can be unambiguously attributed to optical fluctuations, and not to electronic noise, since the evolution of the peak-to-peak voltage value recorded with the oscilloscope reproduces the evolution of the laser average power, which has been recorded, independently, at the same time, with the optical power meter. Fig. 11(c) shows the evolution of the FSR during the 6-h long measurement, yielding a standard deviation of $<0.01 \%$ with respect to its mean value of $36.774 \mathrm{MHz}$. These results show the excellent long-term stability of our allPM resonator. Both short- and long-term high stability of this laser during operation rely on the combination of the laser operation in an all-anomalous-dispersion solitonic regime with an intra-cavity polarizer and a fully-PM configuration.

In order to confirm our initial hypothesis and to check that the slow variations of the pump wavelength are the main

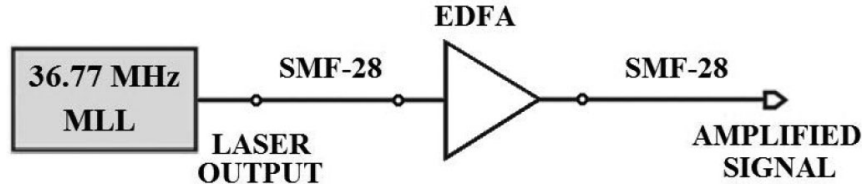

Fig. 13. Experimental setup used for the amplification of the MLL output.

contribution to the measured deviation in the long-term stability, we carried out additional measurements of the average output power of the MLL after incorporating a TEC control to the pumping diode. The diode current and temperature were controlled with a current and temperature controller model Thorlabs CLD1015. Throughout the measurement, the diode was maintained at a constant temperature of $25.00+/-0.01{ }^{\circ} \mathrm{C}$. Fig. 12 shows the evolution of the average output power of the MLL during 6-h long continuous operation after incorporating the TEC control. The inset shows a comparison of the average output power measured for the setup with (black line) and without (red line) the TEC control. The initial standard deviation of $<1.8 \%$ in the average output power measured for the setup without TEC control was reduced down to $0.4 \%$ for the setup with TEC control, confirming our hypothesis.

\section{PULSED AMPLIFICATION AND COMPRESSION}

Stability of the pulsed signal of the laser presented in Section II has also been studied after amplifying the laser output with a standard commercial EDFA IPG EAD-10-C3-P-DC. Fig. 13 shows a scheme of the experimental setup used for the amplification of the pulsed signal. The MLL output was introduced in the EDFA input through a $2.5 \mathrm{~m}$ long SMF-28 passive fiber, and the amplified signal was detected after propagating the EDFA output through a SMF-28 fiber section. The amplifier was tested for a gain range up to $23 \mathrm{~dB}$. The spectrum of the amplified signal remained symmetric up to an amplifier gain of $15 \mathrm{~dB}$. This value was set as a reference to undergo complementary measurements of optical autocorrelation, electric spectrum and phase noise of the photodetected pulses. For this value of the amplifier gain, the amplification process increased the average output power from 0.6 to $20 \mathrm{~mW}$. Over $15 \mathrm{~dB}$, the signal was distorted progressively. As an example, the OS of the amplified signal at $23 \mathrm{~dB}$ gain (with a FWHM bandwidth of $\sim 25 \mathrm{~nm}$ ), is shown in Fig. 14, with an asymmetric profile that indicates pulses presumably shorter but of lower temporal quality.

Fig. 15 shows a comparison of the pulsed signal properties in the optical, temporal and electric domains, measured before (left) and after (right) amplification. Fig. 15(a) and (b), depict the optical spectra measured at the laser output before and after propagating the EDFA output through a $5 \mathrm{~m}$ long section of SMF-28 fiber, respectively. The original $5.9 \mathrm{~nm}$ FWHM optical bandwidth of the laser output was increased to a $15.1 \mathrm{~nm}$ FWHM bandwidth after amplification. Sech ${ }^{2}$ transform-limited nature of the original pulses is not conserved due to optical non-linearities and non-managed dispersion parameters inside the EDFA. Nevertheless, an OS with a symmetric Gaussianlike profile is obtained in a range up to $15 \mathrm{~dB}$ gain operation 


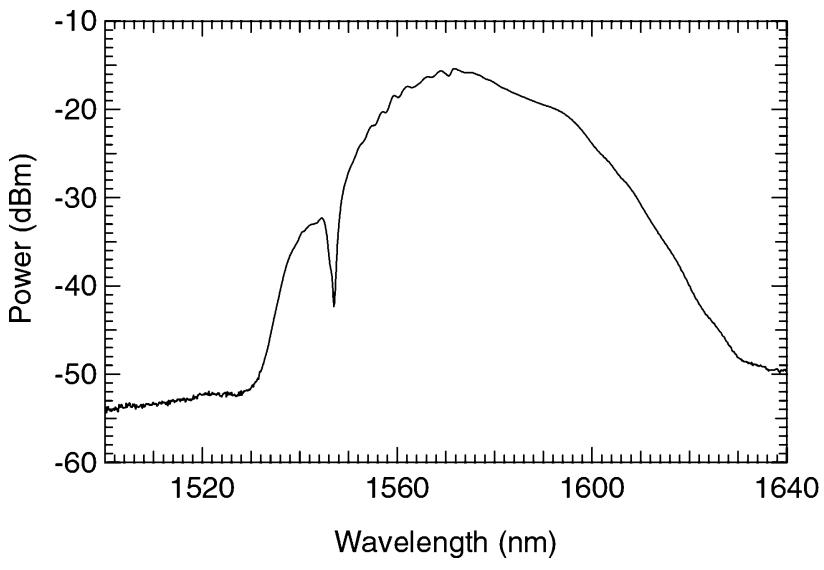

Fig. 14. OS of the amplified signal obtained using an amplifier gain of $23 \mathrm{~dB}$ gain.
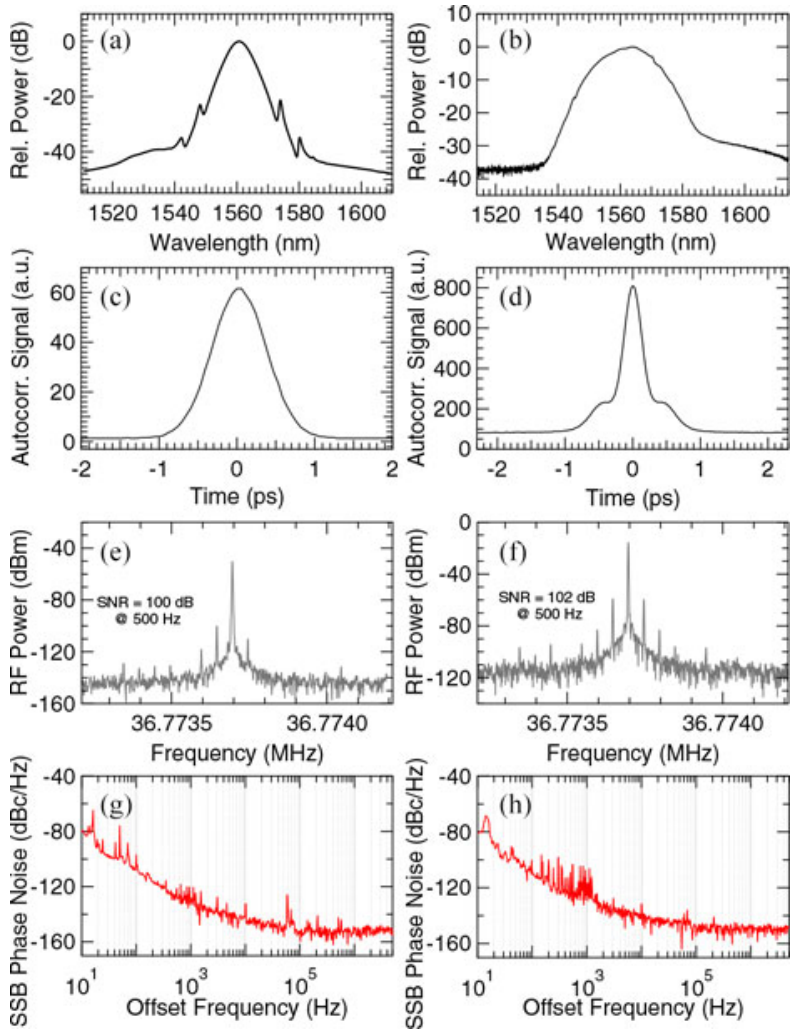

Fig. 15. Comparison of the pulsed signal laser properties before (left) and after (right) amplifying the laser output with a standard commercial EDFA. (a,b) OS, $(\mathrm{c}, \mathrm{d})$ autocorrelation trace, $(\mathrm{e}, \mathrm{f}) \mathrm{RF}$ spectra of the photodetected fundamental harmonic, and $(\mathrm{g}, \mathrm{h})$ phase noise profiles.

of the EDFA. Autocorrelation traces measured before and after amplification are shown in Fig. 15(c) and (d), respectively. The autocorrelation trace presented in Fig. 15(d) was carried out after propagating the amplified signal at the EDFA output through a $52 \mathrm{~m}$ long section of SMF-28 fiber. Such length corresponds to the length required for maximum dispersion-induced compression of the pulses delivered from the EDFA. In this optimum condition, the signal presents a $\sim 244$ fs FWHM pulsewidth. In Fig. 15(e) and (f) we compare the RF spectra of the photodetected fundamental harmonic of the MLL before and after
TABLE II

CAlculated $\Delta \tau$ Time JitTERS FOR DifFERENT $f_{L}$ LOWER FREQUENCY LIMITS BEFORE AND AFTER AMPLIFICATION

\begin{tabular}{|c|c|c|}
\hline \multirow[b]{2}{*}{$\begin{array}{l}\text { Lower integration } \\
\text { limit }\left(f_{L}\right)\end{array}$} & \multicolumn{2}{|c|}{ Time Jitter $(\Delta \tau)$} \\
\hline & $\begin{array}{c}\text { Before } \\
\text { amplification }\end{array}$ & $\begin{array}{c}\text { After } \\
\text { amplification }\end{array}$ \\
\hline $10 \mathrm{~Hz}$ & $3.06 \mathrm{ps}$ & $3.81 \mathrm{ps}$ \\
\hline $100 \mathrm{~Hz}$ & $472.3 \mathrm{fs}$ & $736.9 \mathrm{fs}$ \\
\hline $1 \mathrm{kHz}$ & $414.4 \mathrm{fs}$ & $531.0 \mathrm{fs}$ \\
\hline
\end{tabular}

amplification. As can be seen in this figures, the SNR of $100 \mathrm{~dB}$ measured at a frequency offset of $500 \mathrm{~Hz}$ from the fundamental frequency of the laser output is maintained in the amplified signal. Fig. 15(g) and (h) present the phase noise curves measured at a $f_{c}$ frequency value of $36.77 \mathrm{MHz}$ before and after amplification. If the laser output is amplified before heterodyning it in the PD, the obtained $\Delta \tau$ values are only slightly higher in the amplified signal, as can be seen in Table II.

The numerical tools shown in this paper will be used to design and build amplifiers that maintain the soliton condition of the pulses from the oscillator after amplification.

\section{CONCLUSION}

We demonstrate a short- and long-term operation and environmentally-stable, all-polarization-maintained, FabryPérot cavity, passively mode-locked fiber laser. The cavity design is simple, and it can be scaled to achieve repetition rates in the range of GHz. The combination of the laser operation in an all-anomalous-dispersion solitonic regime with an intracavity polarizer and a fully-PM configuration is demonstrated to be an excellent passive method to obtain both short- and longterm high stability of femtosecond pulse Erbium-doped fiber lasers. Such stability has been studied by a variety of measurements in the temporal and spectral -both optical and electricaldomains. Pulse durations of $540 \mathrm{fs}$, period-relative time jitters of $\sim 0.015 \%$, and long-term standard deviation variations of $<0.01 \%$ in the cavity FSR and $0.4 \%$ in the average output power have been obtained. We have also demonstrated that amplifying the laser output with a conventional EDFA yields measured pulse durations of $\sim 244 \mathrm{fs}$ and an average output power of $20 \mathrm{~mW}$, while maintaining the $100 \mathrm{~dB}$ SNR measured at $500 \mathrm{~Hz}$ offset from the fundamental harmonic frequency of the photodetected signal. The theoretical validation of our experimental results is based on solutions of the NLSE that take into account wavelength and z-position dependence of the active medium gain. When considering such dependences of the active medium gain, numerically calculated values of the emission properties of our fiber laser reproduce the corresponding experimental results with very high accuracy.

\section{REFERENCES}

[1] T. Gherman and D. Romanini, "Mode-locked cavity-enhanced absorption spectroscopy," Opt. Exp., vol. 10, no. 19, pp. 1033-1041, Sep. 2002.

[2] X. Liu, J. Lægsgaard, and D. Turchinovich, "Monolithic highly-stable femtosecond fiber lasers for applications in biophotonics," IEEE J. Sel. Topics Quantum Electron., vol. 18, no. 4, pp. 1439-1450, Jul. 2012. 
[3] J. R. Unruh, E. S. Price, R. G. Molla, L. Stehno-Bittel, C. K. Johnson, and R. Q. Hui, "Two-photon microscopy with wavelength switchable fiber laser excitation," Opt. Exp., vol. 14, no. 21, pp. 9825-9831, Oct. 2006.

[4] Y. M. Lee, R. Y. Tu, A. C. Chiang, and Y. C. Huang, "Average-power mediated ultrafast laser osteotomy using a mode-locked $\mathrm{Nd}: \mathrm{YVO}_{4}$ laser oscillator," J. Biomed. Opt., vol. 12, no. 6, p. 060505, Nov. 2007.

[5] M. Ogusu, K. Inagaki, T. Ohira, I. Ogura, and H. Yokoyama, "Wavelengthdivision multiplexing of two-mode injection-locked Fabry-Prot lasers using optically harmonic modelocked master laser," Electron. Lett., vol. 37, no. 14 , pp. 889-890, Jul. 2001.

[6] A. E. H. Oehler, S. C. Zeller, K. J. Weingarten, and U. Keller, "Broad multiwavelength source with $50 \mathrm{GHz}$ channel spacing for wavelength division multiplexing applications in the telecom C band," Opt. Lett., vol. 33 , no. 18 , pp. $2158-2160$, Sep. 2008.

[7] G. C. Valley, "Photonic analog-to-digital converters," Opt. Exp., vol. 15, no. 5, pp. 1955-1982, Mar. 2007.

[8] F. Laghezza, F. Scotti, P. Ghelfi, A. Bogoni, and S. Pina, "Jitter-limited photonic analog-to-digital converter with 7 effective bits for wideband radar applications," in Proc. Radar Conf., Ottawa, ON, Canada, 2013, pp. $1-5$.

[9] M. E. Fermann and I. Hartl, "Ultrafast fiber laser technology," IEEE J. Sel. Topics Quantum Electron., vol. 15, no. 1, pp. 191-206, Jan. 2009.

[10] H. Byun, M. Y. Sander, A. Motamedi, H. Shen, G. S. Petrich, L. A. Kolodziejski, E. P. Ippen, and F. X. Kärtner, "Compact, stable $1 \mathrm{GHz}$ femtosecond Er-doped fiber lasers," Appl. Opt., vol. 49, no. 29, pp. $5577-$ 5582 , Oct. 2010

[11] A. Martinez and S. Yamashita, "Multi-gigahertz repetition rate passively modelocked fiber lasers using carbon nanotubes," Opt. Exp., vol. 19, no. 7, pp. 6155-6163, Mar. 2011

[12] I. Hartl, G. Imeshev, L. Dong, G. C. Cho, and M. E. Fermann, "Ultracompact dispersion compensated femtosecond fiber oscillators and amplifiers," presented at the Conf. Lasers and Electro-Optics, Baltimore MD, USA, May 22-27, 2005, Paper CThG1.
[13] C. K. Nielsen, B. Ortac, T. Schreiber, J. Limpert, R. Hohmuth, W. Richter, and A. Tunnermann, "Selfstarting self-similar all-polarization maintaining Yb-doped fiber laser," Opt. Exp., vol. 13, no. 23, pp. 9346-9351, Nov. 2005

[14] J. W. Nicholson and M. Andrejco," A polarization maintaining, dispersion managed, femtosecond figure-eight fiber laser," Opt. Exp., vol. 14, no. 18 , pp. 8160-8167, Sep. 2006

[15] N. Nishizawa, Y. Seno, K. Sumimura, Y. Sakakibara, E. Itoga, H. Kataura, and K. Itoh, "All-polarization-maintaining Er-doped ultrashort-pulse fiber laser using carbon nanotube saturable absorber," Opt. Exp., vol. 16 , no. 13, pp. 9429-9435, Jun. 2008.

[16] X. Liu, J. Lægsgaard, and D. Turchinovich, "Highly-stable monolithic femtosecond Yb-fiber laser system based on photonic crystal fibers," Opt. Exp., vol. 18, no. 15, pp. 15475-15483, Jul. 2010.

[17] G. Sobon, J. Sotor, and K. M. Abramski, "All-polarization maintaining femtosecond Er-doped fiber laser mode-locked by Graphene saturable absorber," Laser Phys. Lett., vol. 9, no. 8, pp. 581-586, May 2012.

[18] G. P. Agrawal, Nonlinear Fiber Optics, 4th ed. Boston, MA, USA: Academic, 2007

[19] M. J. F. Digonnet, Rare-Earth-Doped Fiber Lasers and Amplifiers, Revised, and Expanded. New York, NY, USA: CRC Press, 2001.

[20] G. E. Villanueva, M. Ferri, and P. Pérez-Millán, "Active and passive mode-locked fiber lasers for high-speed high-resolution photonic analog to digital conversion," IEEE J. Quantum Electron., vol. 48, no. 11, pp. 1443-1452, Nov. 2012.

[21] G. Serafino, P. Ghelfi, P. Pérez-Millán, G. E. Villanueva, J. Palací, J. L. Cruz, and A. Bogoni, "Phase and amplitude stability of EHF-band radar carriers generated from an active mode-locked laser," IEEE J. Lightw. Technol., vol. 29, no. 23, pp. 3551-3559, Dec. 2011.

Authors' biographies not available at the time of publication. 